\title{
Protective role of microRNA-221 in Parkinson's disease
}

\author{
$\mathrm{Li} \mathrm{L}^{1}, \mathrm{Xu} \mathrm{J}{ }^{2}, \mathrm{Wu} \mathrm{M}{ }^{1}, \mathrm{Hu} \mathrm{JM}^{3}$ \\ Comprehensive Department, The Central Hospital of Wuhan, Wuhan, China. hujunm08@163.com
}

\begin{abstract}
OBJECTIVE: The present study aimed to explore the role and the underlying mechanism of miR-221 in Parkinson's Disease.

MATERIALS AND METHODS: To perform our investigation, a PD cell model was created by using 6-OHDA. Cell viability and proliferation assays, and flow cytometry analysis were performed to detect cell viability and apoptosis. The qRT-PCR and western blotting were used for gene and protein level detection.

RESULTS: We found that the expression of miRNA-221 is significantly lower in 6-OHDA treated PC12 pheochromocytoma cells compared to the normal cells. The results of further analysis indicated that miR-221 mimic significantly promoted the cell viability and proliferation of PC12 cells treated with 6-OHDA. MiR-221 mimic significantly inhibited 6 -OHDA-treated PC12 cells from apoptosis. These effects were eliminated by PTEN over-expression. We also revealed that PTEN was a direct target gene of miR-221. Moreover, we found miR-221 mimic significantly promoted the phosphorylation of AKT in PC12 cells treated with 6-OHDA, and over-expression of PTEN could eliminate this effect. CONCLUSIONS: MiR-221 plays a protective role in Parkinson's Disease via regulating PC12 cell viability and apoptosis by targeting PTEN. Therefore, miR-221 may serve as a potential therapeutic target for Parkinson's disease treatment (Fig. 3, Ref. 27). Text in PDF www.elis.sk.

KEY WORDS: miRNA-221, Parkinson's Disease, PC12 cells, 6-OHDA, PD cell models.
\end{abstract}

\section{Introduction}

Parkinson's disease (PD) is the second most common neurodegenerative disease in the elderly (1) and a progressive neurodegenerative disease manifested by bradykinesia, rigidity, and rest tremor, while up to $80 \%$ of patients eventually develop dementia $(2,3)$. However, a substantial percentage of PD patients have nonmotor symptoms as well. In Sweden, about 20,000 people are affected by PD, with a population of about 9.6 million. Although the neurological basis of cognitive dysfunction in PD is still unclear, the cholinergic system arising from nucleus basalis of Meynert (NBM) located in substantia innominata (SI) of the basal forebrain plays an important role in the cognitive functions of $\mathrm{PD}$ patients (4). Individuals over the age of 60 are twice as likely to develop PD relative to the general population (5).

MicroRNAs (miRNAs) are single-stranded, endogenous noncoding, small ( 22 nucleotides in length) RNAs $(6,7)$. The miRNAs generally bind to the 3 '-untranslated regions (3'-UTRs) of target messenger RNAs (mRNAs) to inhibit gene expression $(8,9)$. They involve many biological processes such as differentiation, apoptosis, proliferation and invasion, and so on (10-12). The miR221 belongs to the miR-221/222 cluster, which locates on the $\mathrm{X}$

${ }^{1}$ Comprehensive Department, The Central Hospital of Wuhan, Wuhan, China, ${ }^{2}$ Department of Orthopedics, The Central Hospital of Wuhan, Wuhan, China, and ${ }^{3}$ Department of Neurosurgery, Wuhan General Hospital of PLA, Wuhan, China

Address for correspondence: JM Hu, Department of Neurosurgery, Wuhan General Hospital of PLA, No. 680 Wuluo Road, Wuhan 430070, China. Phone/Fax: +27.82833081 chromosome and shares some identical seed sequences with its homologous miRNA, miR-222 $(13,14)$. Previous studies have found that miR-221 was significantly up-regulated in several types of human diseases, including hepatocellular carcinoma (15), prostate adenocarcinoma (16), and colorectal carcinoma (17), suggesting its oncogenic role in tumorigenesis and progression. The growth of cancer cells may be significantly different in different cell types, indicating that miR-221 may exhibit promoting cell or keratinocyte formation in different cancer cell lines (18). Therefore, it is important to clarify the exact effect of miR-221 on certain cancer cell lines. However, expression pattern and role of miRNA-221 in the Parkinson's Disease remains poorly understood.

PTEN (Phosphatase and tensin homolog deleted on chromosome 10) is a phosphatase with both protein phosphatase and lipid activities (19). PTEN contains a phosphatase domain, C2 domain, an N-terminal PtdIns, postsynaptic-density protein and C-terminal tail $(20,21)$. The decrease in PTEN function levels is usually associated with elevated activity of PI3K and Akt8 (19). Protein interactions play an important role in the regulation of phosphatase activity of PTEN (22).

In the current study, we explored the roles and the underlying mechanism of miR-221 in Parkinson's disease by gain and loss of function analysis.

\section{Materials and methods}

\section{Reagents}

Rat PCC PC12 cells were acquired from American Type Culture Collection ATCC (Manassas, VA, USA) and cultured in our 
laboratory. Dulbecco's Modified Eagle Medium (DMEM) was acquired from Mediatech (Herndon, VA, USA). Fetal bovine serum (FBS) and 6-OHDA (6-Hydroxydopamine) were acquired from Sigma-Aldrich (St Louis, MO, USA). All primary antibodies (anti$\beta$-actin, anti-pAKT, anti-PTEN, anti-AKT) were acquired from Cell Signaling Technology (Boston, MA, USA).

\section{Cell culture and treatment}

PC12 cells were cultured in Dulbecco's modified Eagle's medium (DMEM) supplemented with $10 \%$ fetal bovine serum, $100 \mathrm{U} \mathrm{ml}-1$ penicillin and $100 \mathrm{U} \mathrm{ml-1}$ streptomycin $(\mathrm{pH} 7.2)$ in a $5 \% \mathrm{CO}_{2}$ atmosphere at $37^{\circ} \mathrm{C}$. 6-OHDA was dissolved in sterile distilled water to prepare the stock solutions ( $2 \mathrm{ug} / \mathrm{ul})$, aliquoted and stored at $-80^{\circ} \mathrm{C}$.

PC12 cells were treated as follows. 1) Cells were treated with or without 100uM 6-OHDA for $24 \mathrm{~h}$ at $37{ }^{\circ} \mathrm{C}, 5 \% \mathrm{CO}_{2}$. 2) Cells were transiently transfected with miR-221 mimic for $48 \mathrm{~h}$ at 37 ${ }^{\circ} \mathrm{C}, 5 \% \mathrm{CO}_{2}$. They were treated with or without $100 \mathrm{uM} 6$-OHDA for $24 \mathrm{~h}$ at $37{ }^{\circ} \mathrm{C}, 5 \% \mathrm{CO}_{2}$. 4) Cells were transiently transfected with miR-221 mimic and PTEN plasmid for $48 \mathrm{~h}$ at $37^{\circ} \mathrm{C}, 5 \%$ $\mathrm{CO}_{2}$. Then they were analyzed by western blotting, qRT-PCR, cell viability and proliferation assays and flow cytometry.

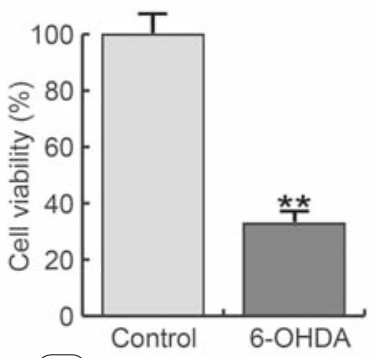

(A)
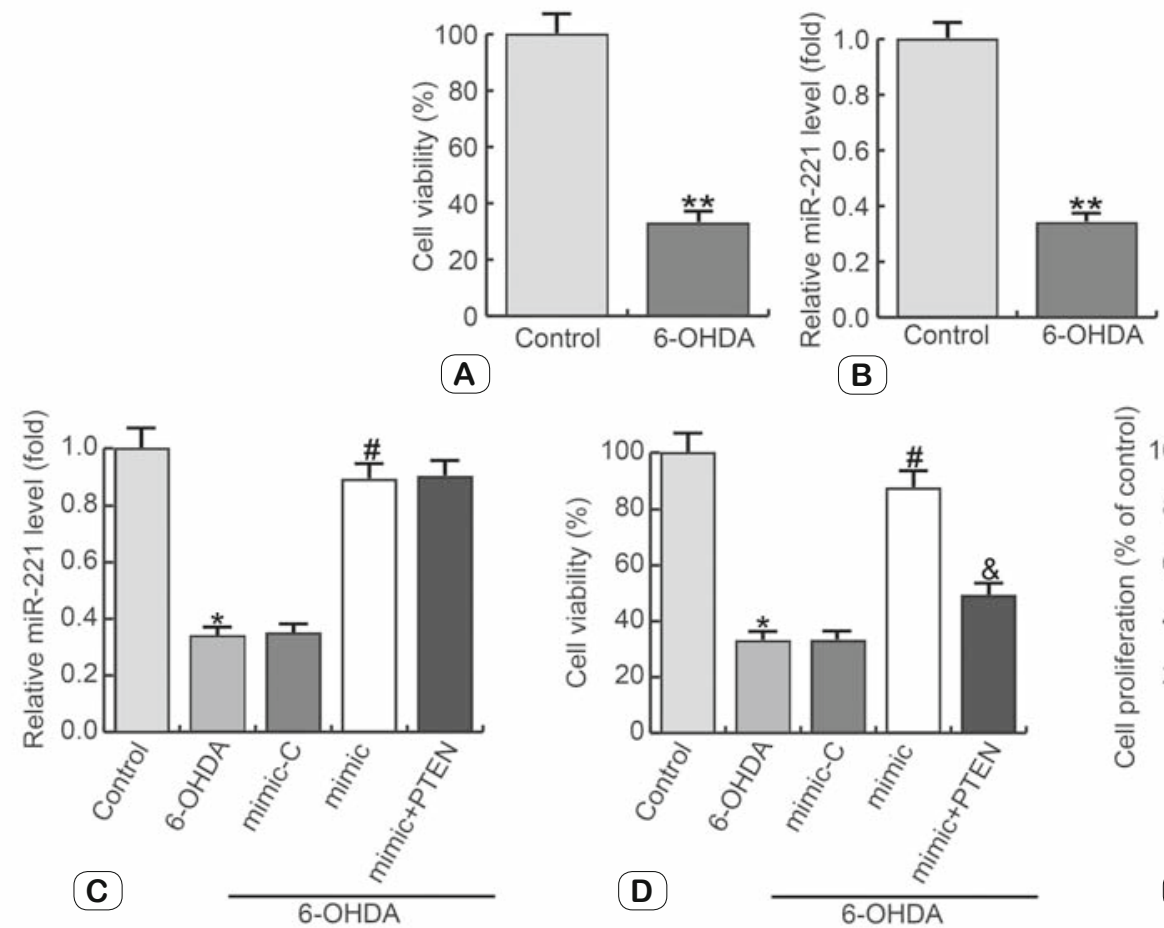

B reaction $(q R T-P C R)$

Total RNA was extracted from the PC12 cells and stored in liquid nitrogen according to instructions from the RNA extraction kit (Z3100, Promega, Madison, WI, USA). For RNA quantification, ratio of OD values at $260 \mathrm{~nm}$ and $280 \mathrm{~nm}$ (A260/A280) were determined for all samples by an ultraviolet spectrophotometer. The RNA samples were stored at $-80^{\circ} \mathrm{C}$ for future use. The firststrand cDNA was synthesized by M-MLV Reverse Transcriptase (Promega) with oligo(dT) primers. The QuantiFast SYBR Green PCR Kit (Qiagen) was applied for quantitative real-time PCR (qRT-PCR) with a CFX Connect Real-Time System (Bio-Rad). Target quantifications were performed with specific primer pairs designed using Primer 5 software.

\section{Western blot analysis}

After treatment, the cell culture solution was removed, first with pre-cooling PBS rinse 3 times. On ice, they were lysed in RIPA buffer (50 mM Tris, $\mathrm{pH} 7.2 ; 1 \%$ Triton-X 100; $1 \%$ sodium deoxycholate; $1 \mathrm{mM} \mathrm{Na}_{3} \mathrm{VO}_{4} ; 150 \mathrm{mM} \mathrm{NaCl} ; 10 \mathrm{mM} \mathrm{NaF} ; 0.1 \%$ SDS; protease inhibitor cocktail $(1: 1000))$. The lysate was sonicated for 10 seconds and centrifuged at $13,000 \mathrm{~g}$ for 10 minutes

Fig. 1. miR-221 over-expression reversed the effects of 6-OHDA on PC12 cell survival and proliferation. miR-221 expression is down-regulated in 6-OHDA-treated PC12 cells. A - 6-OHDA $(100 \mu \mathrm{M})$ treatment decreased PC12 cell viability. Cell viability was measured by MTT assay; B - qRT-PCR assay was used to determine the level of miR-221; C - The level of miR-221 in different groups was determined by qRT-PCR assay; D - miR-221 significantly increased 6-OHDA $(100 \mu \mathrm{M})$ induced PC12 cell death. Cell viability was measured by MTT assay; E - PC12 cell proliferation was measured by $\mathrm{CCK}-8$ assay. Control - cells without any treatment; 6-OHDA - cells treated with 6-OHDA (100 $\mu$ M) for $24 \mathrm{~h}$; mimic-C - cells transfected with the control of miR-221 mimic for $48 \mathrm{~h}$ and then treated with 6-OHDA (100 $\mu \mathrm{M})$ for $24 \mathrm{~h}$; mimic - cells transfected with miR-221 mimic for $48 \mathrm{~h}$ and then treated with 6-OHDA $(100 \mu \mathrm{M})$ for $24 \mathrm{~h}$; mimic+PTEN - cells co-transfected with miR-221 mimic and PTEN-plasmids for $48 \mathrm{~h}$ and then treated with 6-OHDA (100 $\mu \mathrm{M})$ for $24 \mathrm{~h}$. Experiments were repeated three times. Data are presented as mean \pm SD. *, ** $p<0.05,0.01$ vs control; \# $p<0.05$ vs 6 -OHDA; \&p $<0.05$ vs mimic. 
at $4{ }^{\circ} \mathrm{C}$. The protein concentration was determined by quinolinic acid using bovine serum albumin as a standard. An equal amount of protein was isolated on a 7.5-12\% SDS-polyacrylamide gel and transferred onto a polyvinylidene difluoride membrane. The PVDF membrane was treated with methanol for 30-60 seconds and placed in transfer buffer before being used. The membranes were incubated with PBS containing $0.05 \%$ Tween 20 and $5 \%$ skim milk to block nonspecific binding, incubated with the primary antibody and then incubated with the appropriate secondary antibody conjugated with horseradish peroxidase Rabbit IgG goat polyclonal antibody). The band density was quantified using Gel-Pro Analyzer optical density software (Media Cybernetics).

\section{Cell viability and proliferation assays}

To detect cell viability, MTT assay was performed after specific treatment. The cells were evenly inoculated into 96-well plates (Corning Costar, Corning, NY, USA) with $2.0 \times 10^{3}$ cells per well. MTT solution was added at indicated times, $20 \mu \mathrm{l}$ per well, and then incubated for $4 \mathrm{~h}$ at $37^{\circ} \mathrm{C}$. After removal of the supernatant, dimethyl sulfoxide (DMSO; Sigma) was added at $200 \mu$ l per well. The absorbance at $490 \mathrm{~nm}$ was measured by a microplate reader for cell viability calculation. Meanwhile, to determined PC12 cell proliferation, cell counting kit-8 (CCK-8 kit, Beyotime Biotechnology, Jiangsu, China) was applied according to the manufacturer's instructions.

\section{Flow cytometry analysis}

After specific treatment, cells were collected and washed with PBS (phosphate-buffered saline). Then cell apoptosis analysis was performed. In brief, cells $\left(1 \times 10^{6}\right)$ from each sample were re-suspended in binding buffer, and stained with the labeled with annexin V-FITC and propidium iodide (PI) using an AnnexinVFITC/PI kit (BD Pharmingen, San Diego, CA, USA) according to the manufacturer's instructions. At the end of the experiment, cells were analyzed and quantified by flow cytometry (BD FACS Aria; BD Biosciences, Franklin Lakes, NJ, USA).

\section{Dual luciferase reporter analysis}

Potential target genes of miR-221, including PTEN, were selected by using microRNA target site prediction software. To explore whether miR-221 targets the 3'-UTRs of PTEN, a cDNA fragment of the PTEN-3'-UTR mRNA containing the seed sequence of the mature miR-221 binding site or a mutated binding site of the 3'-UTR sequence was cloned into the pmirGLO dualluciferase vector (Promega, Madison, WI, USA), and the vectors named PTEN-3'UTR-WT and PTEN-3'UTR-MUT, respectively. PC12 cells were seeded into a 24-well plate and then co-transfected with PTEN-3'UTR-WT or PTEN-3'UTR-MUT and miR-221 or its control (hsamiR-C) vector. Luciferase activity was assessed by performing a Dual-Luciferase Reporter Assay Kit (Promega, USA) in line with the manufacturer's protocols. All Tests were repeated at least 3 times.

\section{Statistical analysis}

Data were displayed as mean values \pm SEM. One-way ANO-
VAs or Student's t tests was used for comparisons between groups. A level of $\mathrm{P}<0.05$ was considered to be significant.

\section{Results}

\section{Down-regulation of miR-221 in PD}

We used PC 12 cells treated with 100 uM 6-OHDA to create PD cell models. Compared with the control group, the viability of PC12 cells treated with 100 uM 6-OHDA were significantly decreased. Previous studies have shown that miR-221 is significantly lower in the serum of Parkinson's patients. Our findings suggest that miR-221 is significantly lower in the $100 \mathrm{uM}$ 6-OHDA-induced PD cell model, consistent with previous studies.

\section{MiR-221 promotes PC12 cell viability and proliferation}

To evaluate the biological effects of miR-221 in PD cell models, PC12 cells were transiently transfected with miR-221 mimic, mimic-control, or miR-221 mimic + PTEN plasmid for $48 \mathrm{~h}$, and the transfection efficiency was determined by qRT-PCR. Then we performed the viability and proliferation assay. The results indicated that the viability and proliferation of 6-OHDA-treated PC12 cells were significantly promoted by miR-221 mimic, compared to control group and mimic-control group. The over-expression of PTEN could eliminate the effect of miR-221 mimic on the viability and proliferation of 6-OHDA treated PC12 cells.

\section{MiR-221 inhibits PC12 cell apoptosis}

The effect of miR-221 on PC12 cell apoptosis was determined. As shown in Figure 1A and B, the 6-OHDA-treatment induced PC12 cell apoptosis were significantly inhibited by miR-221 mimic. And in the PTEN over-expression group, the effect of miR-221 mimic on the apoptosis of PD cell models was eliminated.

\section{PTEN is a direct target of miR-221}

By using microRNA target site prediction software, we identified a putative miR-221 binding site located in the 3'-UTR of PTEN mRNA (Fig. 3A). Then we performed the dual-luciferase reporter assay to directly confirm whether miR-221 binds to PTEN. We found that miR-221 significantly suppressed the luciferase activity of the wild-type PTEN 3'UTR (WT) but not Mut 3'-UTR of PTEN in PC12 cells (Fig. 3B). Besides, to confirm this relationship, we further determined the effect of miR-221 on PTEN expression in PC12 cells. The qRT-PCR analysis demonstrated that PTEN mRNA expression was inhibited after transfection of miR-221 mimic in PD cell models (Fig. 3C). Similarly, PTEN protein level was also decreased in cells transfected with miR221 mimic (Fig. 3D). Taken together, miR-221 can directly target PTEN in PD cell models.

\section{Effect of miR-221 on PI3K/AKT pathway}

The effect of miR-221 on PI3K/AKT pathway was investigated in the present study. The results suggested that the phosphorylation of AKT in PC12 cells were significantly decreased by 6-OHDA treatment. Compared to control group and mimic-control group, miR-221 mimic significantly promoted p-AKT expression in PC12 

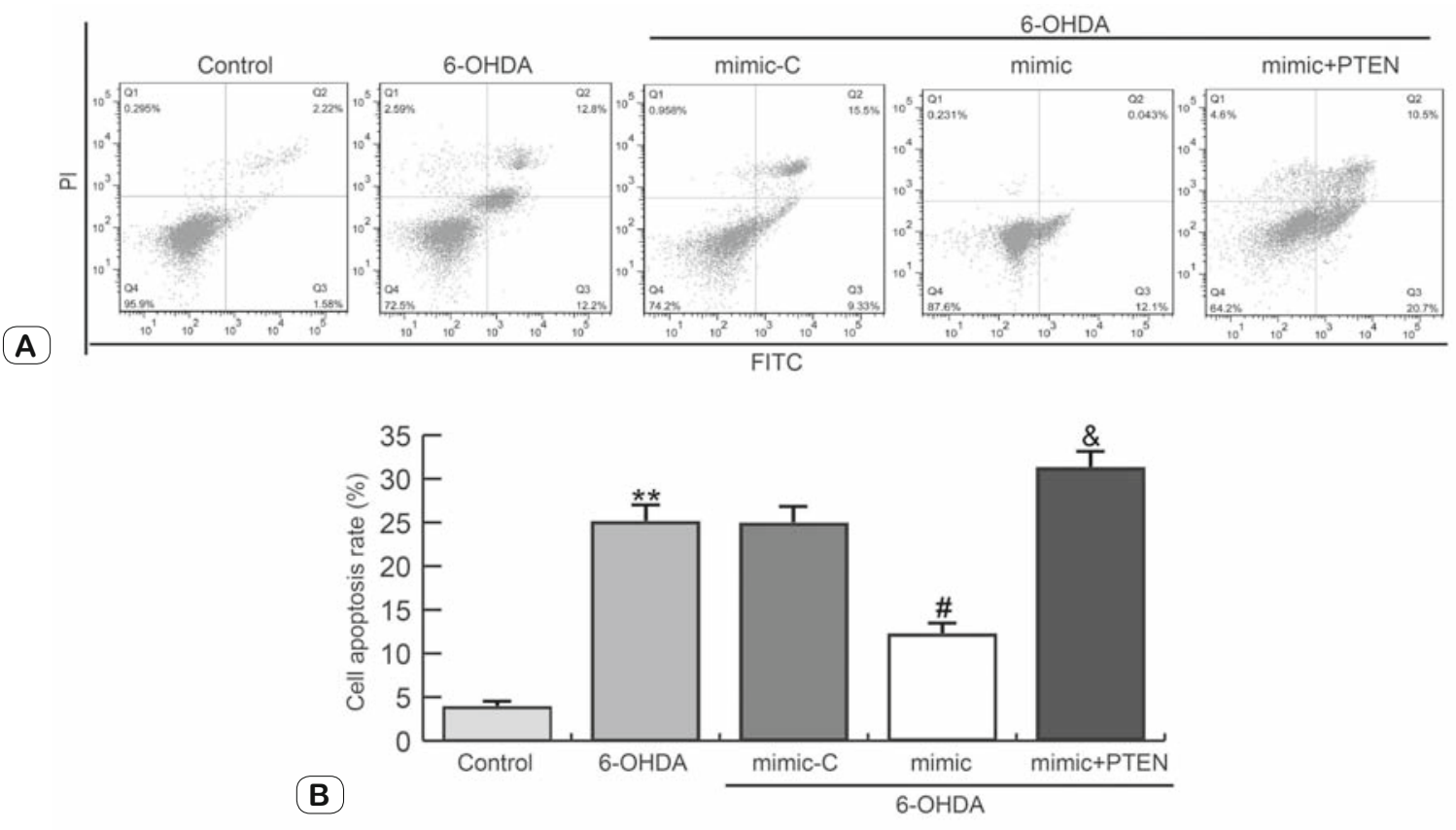

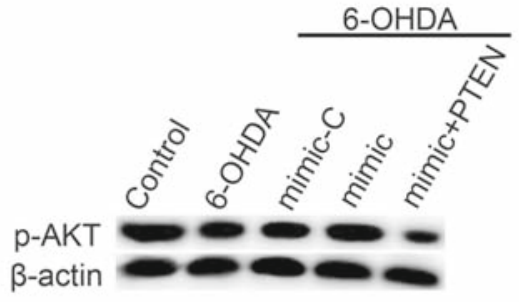

(C)

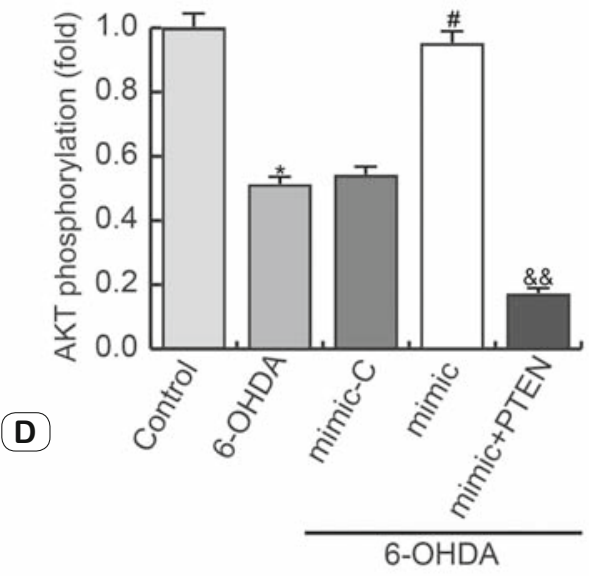

Fig. 2. miR-221 over-expression reversed the effects of 6-OHDA on PC12 cell apoptosis. PC 12 cell apoptosis in different groups was determined by FCM (A), and data was analyzed (B). miR-221 over-expression reversed the effects of 6-OHDA on p-AKT expression in PC12 cells. $\mathrm{C}$ - protein level of $\mathrm{p}$-AKT was detected by western blotting; D - the expression levels of AKT phosphorylation were expressed fold of the control.Control - cells without any treatment; 6-OHDA - cells treated with 6-OHDA (100 $\mu \mathrm{M})$ for $24 \mathrm{~h}$; mimic-C - cells transfected with the control of miR-221 mimic for $48 \mathrm{~h}$ and then treated with 6-OHDA $(100 \mu \mathrm{M})$ for $24 \mathrm{~h}$; mimic - cells transfected with miR-221 mimic for $48 \mathrm{~h}$ and then treated with 6-OHDA $(100 \mu \mathrm{M})$ for $24 \mathrm{~h}$; mimic+PTEN - cells co-transfected with miR-221 mimic and PTEN-plasmids for $48 \mathrm{~h}$ and then treated with 6-OHDA $(100 \mu \mathrm{M})$ for $24 \mathrm{~h}$. Experiments were repeated three times. Data are presented as mean $\pm \mathrm{SD}$. *,** p $<0.05,0.01 \mathrm{vs}$ control; \# $\mathrm{p}<0.05$ vs $6-\mathrm{OHDA} ; \&, \& \& \mathrm{p}<0.05,0.01$ vs mimic.

cells treated with 6-OHDA. In the PTEN over-expression group, the effect of miR-221 mimic on p-AKT expression in PC12 cells was eliminated. The expression of total AKT was not significantly different between groups (Figs $1 \mathrm{C}$ and D).

\section{PTEN is a direct target of miR-221}

By using microRNA target site prediction software, we identified a putative miR-221 binding site located in the 3'-UTR of PTEN mRNA (Fig. 2A). Then we performed the dual-lucifer- ase reporter assay to directly confirm whether miR-221 binds to PTEN. We found that miR-221 significantly suppressed the luciferase activity of the wild-type PTEN 3'UTR (WT) but not Mut 3'-UTR of PTEN in PC12 cells (Fig. 2B). Besides, to confirm this relationship, we further determined the effect of miR-221 on PTEN expression in PC12 cells. qRT-PCR analysis demonstrated that PTEN mRNA expression was inhibited after transfection of miR-221 mimic in PD cell models (Fig. 2C). Similarly, PTEN protein level was also decreased in cells transfected with miR- 

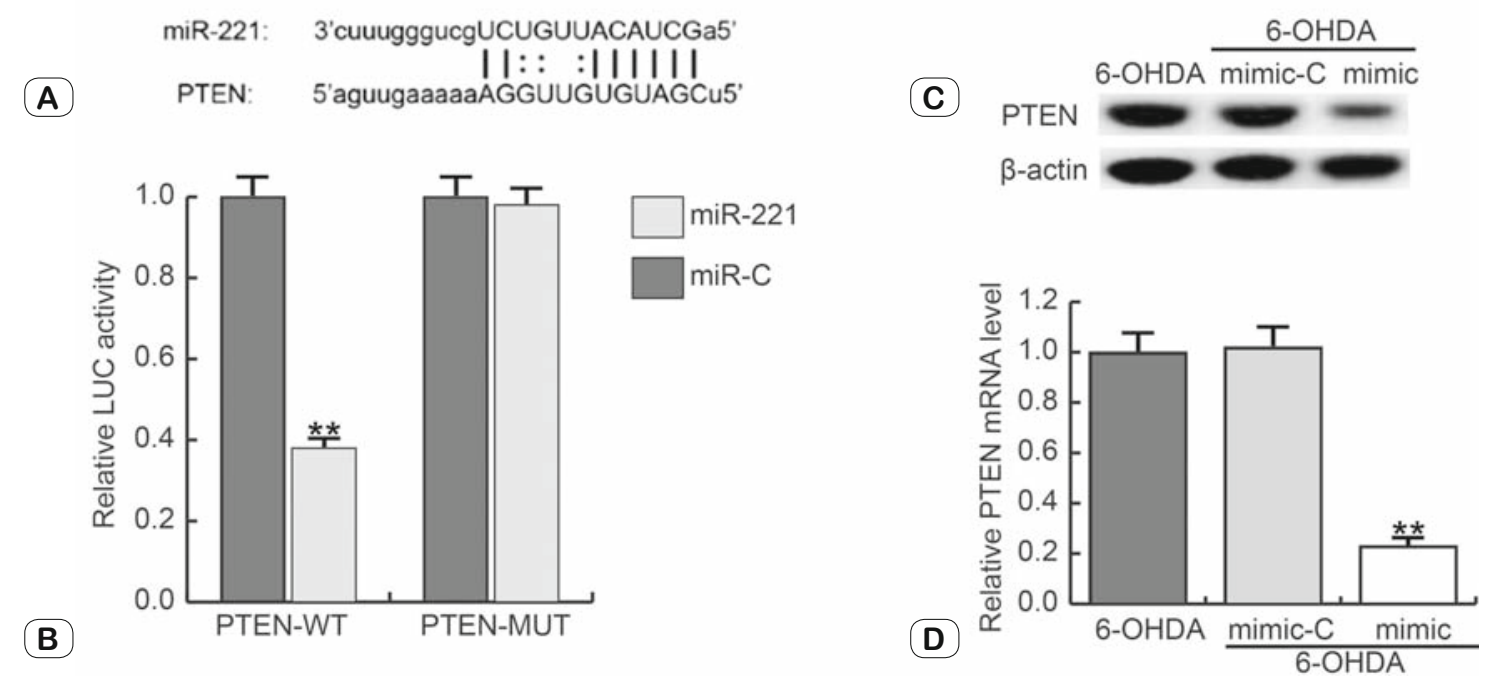

Fig. 3. PTEN is a direct target of miR-221. A - Interaction between miR-221 and 3'UTR of PTEN was predicted using microRNA target site prediction software; B - Luciferase activity of a reporter containing a wild-type PTEN 3'UTR or a mutant PTEN 3' UTR are presented. "PTEN3'UTR-MUT'" indicates the PTEN 3' UTR with a mutation in the miR-221 binding site. UTR, untranslated region. All data are presented as the mean \pm SD of three independent experiments. ** $p<0.01$ vs control. C - protein level of PTEN was detected by western blotting; D - mRNA level of PTEN was measured by qRT-PCR. 6-OHDA - cells treated with 6-OHDA $(100 \mu \mathrm{M})$ for $24 \mathrm{~h}$; mimic-C - cells transfected with the control of miR-221 mimic for $48 \mathrm{~h}$ and then treated with 6-OHDA $(100 \mu \mathrm{M})$ for $24 \mathrm{~h}$; mimic - cells transfected with miR-221 mimic for $48 \mathrm{~h}$ and then treated with 6-OHDA $(100 \mu \mathrm{M})$ for $24 \mathrm{~h}$. Experiments were repeated three times. Data are presented as mean \pm SD. $* *$ p $<0.01$ vs 6-OHDA.

221 mimic (Fig. 2D). Taken together, miR-221 can directly target PTEN in PD cell models.

\section{Discussion}

Although the molecular mechanisms of some miRNAs are understood in the process of Parkinson's disease, specific patterns of miRNA expression in Parkinson's disease development still remained poorly understood. Therefore, it is necessary to thoroughly investigate the function of miRNAs specifically involved in Parkinson's disease and develop a new regulating role of miR-221 in the malignant progression of Parkinson's disease.

In the current study, we explored the roles and the underlying mechanism of miR-221 in Parkinson's disease by gain and loss of function analysis. We showed that MiR-221 was low in expression in Parkinson's syndrome. When miR-221 is highly expressed, it promotes cell proliferation and inhibits cell apoptosis. Thus, it is important to elucidate the exact effect of miR-221 on Parkinson's disease.

First of all, we found that the expression of miR-221 was down-regulated in PD cell models. This result suggests that miR221 participates in Parkinson's Disease. Previous studies have indicated that miRNA abnormal expression is involved in various biological processes $(18,23)$. To reveal the role of miR-221 in PD cell models, we tested the effect of miR-221 on cell growth, and we showed that increased miR-221 expression could promote cell proliferation and inhibits cell apoptosis.

The miRNAs generally carry out biological functions by directly binding to the 3'-UTR of mRNAs. We primarily determine that PTEN was the predictive target of miR-221 by using the TargetScan. Luciferase activity assay suggested direct targeting of PTEN by miR-221. Furthermore, we also found that the expression of miR-221 negatively regulated PTEN expression in PD cell models. QRT-PCR and western blot analyses confirmed that PTEN expression was significantly decreased in PC cells transfected with PTEN mimic, compared to those transfected with the corresponding negative control. These results suggested that PTEN was a target of miR-221 in Parkinson's Disease.

PTEN is a multifaceted tumor suppressor and extremely powerful functionally involved in many different forms of cancer. PTEN acts as a tumor suppressor mainly involved in the homeostatic maintenance of the phosphatidylinositol 3 kinase (PI3K)/ AKT cascade (24), and PTEN is also the most important negative regulator of the PI3K signaling pathway. In addition to its canonical PI3K inhibition-dependent functions, PTEN can also function as a multifaceted tumor suppressor in the PI3K-independent manner (25). The main mechanism by which PTEN activity restrains cancer development and progression remains in its ability to down-modulate the signaling through PI3K pathway, thereby indirectly inhibiting AKT downstream targets such as FOXO, GSK3 $(26,27)$. We recently demonstrated that the PI3K-Akt signaling pathway was up-regulated by miR-221. But in the PTEN over-expression group, the effect of miR-221 mimic on p-AKT expression in PC12 cells was eliminated. Over-expression of PTEN may inhibit cell proliferation and promote apoptosis by inhibiting PI3K/AKT pathway.

Taken together, we found for the first time that miR-221 played a protective role in Parkinson's disease. Therefore, miR-221 may 
serve as a potential therapeutic target for the treatment of Parkinson's disease.

\section{References}

1. Ran C, Wirdefeldt K, Brodin L et al. Genetic Variations and mRNA Expression of NRF2 in Parkinson's Disease. Parkinsons Dis 2017; 4020198.

2. Postuma RB, Berg D, Stern M et al. MDS clinical diagnostic criteria for Parkinson's disease. Mov Disord 2015; 30: 1591-1601.

3. Hely MA, Reid WG, Adena MA et al. The Sydney multicenter study of Parkinson's disease: the inevitability of dementia at 20 years. Mov Disord 2008; 23: 837-844.

4. Kim I, Shin NY, Yunjin Bak et al. Early-onset mild cognitive impairment in Parkinson's disease: Altered corticopetal cholinergic network. Sci Rep 2017; 7: 2381.

5. Powers R, Lei S, Anandhan A et al. Metabolic Investigations of the Molecular Mechanisms Associated with Parkinson's Disease. Metabolites $2017 ; 7$.

6. Victor A. The functions of animal microRNAs. Nature 2004; 431 : 350-355.

7. Thalia AF, Jessica IH, Pavel M et al. MicroRNAs in human cancer. MicroRNA Cancer Regulation pp 1-20, 2012.

8. Schickel R, Boyerinas B, Park SM et al. MicroRNAs: key players in the immune system, differentiation, tumorigenesis and cell death. Oncogene 2008; 27: 5959-5974.

9. Takasaki S. Roles of microRNAs in cancers and development. Methods Mol Biol 2015; 1218: 375-413.

10. Bartel DP. MicroRNAs: genomics, biogenesis, mechanism, and function. Cell 2004; 116: 281-297.

11. George AC, Carlo MC. MicroRNA signatures in human cancers. Nature Rev Cancer 2006; 6: 857-866.

12. Li J, Wang Y, Song $\mathbf{Y}$ et al. miR-27a regulates cisplatin resistance and metastasis by targeting RKIP in human lung adenocarcinoma cells. Mol Cancer 2014; 13: 193.

13. Chen WX, Hu Q, Qiu MT et al. miR-221/222: promising biomarkers for breast cancer. Tumour Biol 2013; 34: 1361-1370.
14. Liu S, Sun X, Wang M et al. A microRNA 221-and 222-mediated feedback loop maintains constitutive activation of NFkappaB and STAT3 in colorectal cancer cells. Gastroenterology 2014; 147: 847-859.

15. Liu M, Liu J, Wang L et al. Association of serum microRNA expression in hepatocellular carcinomas treated with transarterial chemoembolization and patient survival. PLoS One 2014; 9: e109347.

16. Zheng Q, Peskoe SB, Ribas $\mathbf{J}$ et al. Investigation of miR-21, miR141 , and miR-221 expression levels in prostate adenocarcinoma for associated risk of recurrence after radical prostatectomy. Prostate 2014; 74 : $1655-1662$.

17. Tao K, Yang J, Guo $\mathbf{Z}$ et al. Prognostic value of miR-221-3p, miR342-3p and miR-491-5p expression in colon cancer. Am J Transl Res 2014; 6: $391-401$.

18. Yin Z, Xu M, Li P. miRNA-221 acts as an oncogenic role by directly targeting TIMP2 in non-small-cell lung carcinoma. Gene 2017; 620: 46-53.

19. Song MS, Salmena L, Pandolfi PP. The functions and regulation of the PTEN tumour suppressor. Nat Rev Mol Cell Biol 2012; 13: 283-296.

20. Leslie NR, Batty IH, Maccario $\mathrm{H}$ et al. Understanding PTEN regulation: PIP2, polarity and protein stability. 2008; 27: 5464-5476.

21. Wu Q, Li Z, Mellor P et al. The role of PTEN - HCV core interaction in hepatitis C virus replication. Sci Rep 2017; 7: 3695.

22. Milella M, Falcone I, Conciatori F et al. PTEN: Multiple Functions in Human Malignant Tumors. Front Oncol 2015; 5: 24.

23. Quann K, Jing Y, Rigoutsos I. Post-transcriptional regulation of BRCA1 through its coding sequence by the miR-15/107 group of miRNAs. Front Genet 2015; 6: 242.

24. Manning BD, Cantley LC. AKT/PKB signaling: navigating downstream. Cell 2007; 129: 1261-1274.

25. Tamura M, Gu J, Matsumoto $\mathbf{K}$ et al. Inhibition of cell migration, spreading, and focal adhesions by tumor suppressor PTEN. Science 1998; 280:1614-1617.

26. Ciuffreda L, Di Sanza C, Incani UC et al. The mTOR pathway: a new target in cancer therapy. Curr Cancer Drug Targets 2010; 10: 484-495.

27. Ma XM, Blenis J. Molecular mechanisms of mTOR-mediated translational control. Nat Rev Mol Cell Biol 2009; 10: 307-318.

Received August 3, 2017. Accepted September 8, 2017. 